\section{Bacteremic meningitis due to Pasteurella multocida resistant to first line antibiotic therapy}

\author{
Damiano Larnè, ${ }^{1}$ Manuela Ceccarelli, ${ }^{1}$ \\ Fabrizio Condorelli, ${ }^{2}$ \\ Emmanuele Venanzi Rullo, ${ }^{1}$ \\ Giuseppe Nunnari, \\ Giovanni Francesco Pellicanò ${ }^{3}$ \\ ${ }^{1}$ Department of Clinical and \\ Experimental Medicine, Unit of \\ Infectious Diseases, University of \\ Messina; ${ }^{2}$ Department of \\ Pharmacological Sciences, Università del \\ Piemonte Orientale "A. Avogadro", \\ Novara; ${ }^{3}$ Department of Human \\ Pathology of the Adult and the \\ Developmental Age “G. Barresi”, Unit of \\ Infectious Diseases, University of \\ Messina, Italy
}

\begin{abstract}
Pasteurella species reside in the gastrointestinal tract of many animals, especially in pets such as cats or dogs. Zoonotic transmission of Pasteurella to human is documented. We describe a case of meningitis in a 66-year-old woman with positive blood culture for Pasteurella multocida. Meningitis caused by zoonosis agents is a rare event, but it should be suspected in patients that have recreational or professional exposure to animals. In this case, not only the etiologic agent was rare, but the microorganism was also resistant to firstline antibiotic drugs.
\end{abstract}

\section{Introduction}

Pasteurella species are facultative anaerobic gram-negative coccobacilli, normally found in the oral cavity and upper respiratory tract of the animal population. Transmission to humans is possible through animal bites, wounds or inhalation route.

Pasteurella multocida is the species most frequently involved in human infections. ${ }^{2}$ The principal clinical manifestation of pasteurellosis is represented by local skin infections following animal bites or scratches, but it is also possible to observe pneumonia, bacteremia, osteomyelitis, endocarditis and meningitis. ${ }^{1}$

During the last 65 years, approximately 100 cases of Pasteurella multocida meningitis were reported, especially in a pediatric setting.

Hereby we describe a case of an unusu- al and severe clinical manifestation of Pasteurella multocida infection with documented resistance to first-line therapy.

\section{Case Report}

We describe a case of a 66-year-old woman admitted to the Infectious Disease ward of the G. Martino University Hospital, in Messina (Italy), for fever arisen 24 hours before, with nucal rigor, mental confusion and right earache.

Laboratory investigation revealed an increase of C Reactive Protein (CRP) (8.70 $\mathrm{mg} / \mathrm{dL}$; normal $<0.50 \mathrm{mg} / \mathrm{dL}$ ) and neutrophilic leukocytosis. A head computerized tomography (CT) scan highlighted mastoiditis and otitis with pathological tissue around the right ossicular chain. In the suspicion of meningitis, we performed blood cultures and tried to execute a lumbar puncture. The attempt was not successful due to the obese habitus of the patient.

We then started a therapy with corticosteroids (dexamethasone $0.15 \mathrm{mg} / \mathrm{kg}$ q $6 \mathrm{~h}$ for three days) and an empiric antibiotic treatment with ceftriaxone $2 \mathrm{~g}$ bid and ampicillin/sulbactam $2 / 1 \mathrm{~g}$ qid. On the $4^{\text {th }}$ day of the admission, blood culture resulted positive for Pasteurella multocida (identified with Vitek 2.0, then confirmed with Maldi Tof), resistant to ampicillin and cephalosporins (Table 1). On the other hand, it was susceptible to rifampin, levofloxacin and trimethoprim/sulfamethoxazole (TMP/SMX); therefore, we changed the antibiotic therapy, stopping ampicillin/sulbactam and ceftriaxone and starting rifampin $600 \mathrm{mg}$ qd, levofloxacin $750 \mathrm{mg}$ qd and $1280 / 6400 \mathrm{mg}$ of intravenous TPM/SMX divided in four doses. During her staying in our ward, the patient's conditions gradually improved, and we discharged her on the $12^{\text {th }}$ day after admission in good clinical conditions.

Only after the isolation of Pasteurella multocida in blood cultures, when the patient was in better health and able to report about her risk factors, she told us she was used to feed many stray cats living near her home.

\section{Informed consent}

The patient signed a standard form of consent, expressing her informed consent for the scientific use of her clinical data.

\section{Discussion}

The principal clinical manifestation of pasteurellosis is represented by local skin
Correspondence: Manuela Ceccarelli, Department of Clinical and Experimental Medicine, Unit of Infectious Diseases, University of Messina, Via Consolare Valeria, 1, 98125 Messina (ME), Italy.

Tel.: +39.090.221.3667.

E-mail: manuela.ceccarelli86@gmail.com

Key words: zoonosis, bacteremia, Pasteurella multocida, meningitis.

Acknowledgements: the authors would like to thank the whole Unit of Infectious Diseases of the University Hospital "G. Martino" of Messina for their kind cooperation.

Contributions: DL and MC wrote the article; FC and EVR searched the literature; GN and GFP revised the manuscript.

Conflict of interest: the authors declare no potential conflict of interest.

\section{Funding: none.}

Received for publication: 6 February 2018.

Revision received: 28 March 2018.

Accepted for publication: 14 April 2018.

This work is licensed under a Creative Commons Attribution-NonCommercial 4.0 International License (CC BY-NC 4.0).

(C) Copyright D. Larnè et al., 2018

Licensee PAGEPress, Italy

Infectious Disease Reports 2018; 10:7632

doi:10.4081/idr.2018.7632

infections following animal bites or scratches.

We report a case of meningitis caused by Pasteurella multocida in an immunocompetent patient. Although meningitis is an uncommon outcome of a $P$. multocida infection, it has been reported in the past, especially in a pediatric population, making this micro-organism a rare cause of adult bacterial meningitis. ${ }^{1,3}$ Furthermore, studies have shown a mortality ranging from 7 to $31 \%$ in patients with Pasteurella bacteremia. $^{4}$

The source of infection in our patient was probably represented by the stray cats she fed, but she could not remember any scratch or bite. Literature reports that the main risk factor for Pasteurella multocida meningitis is represented by contact with animals, which is suggested as a source of infection in $84 \%$ of cases. ${ }^{5}$ Transmission can also occur through contact with the animal's saliva. ${ }^{6}$ Another risk factor is an age over 55 years old. ${ }^{5}$ Our patient was a 66year-old woman and a cat-owner; thus, she was at high-risk for this form of meningitis. 
Table 1. Blood culture results and susceptibility testing for Pasteurella multocida. Result: positive. Micro-organism: Pasteurella multocida.

\begin{tabular}{ll}
\hline Antibiotic & Sensitive (S), Intermediate (I), Resistant (R) \\
Amikacin & $\mathrm{S}$ \\
Ampicillin & $\mathrm{R}$ \\
\hline Cefepime & $\mathrm{R}$ \\
Cefotaxime & $\mathrm{R}$ \\
\hline Ceftazidime & $\mathrm{R}$ \\
Ciprofloxacin & $\mathrm{S}$ \\
\hline Gentamicin & $\mathrm{S}$ \\
Imipenem & $\mathrm{R}$ \\
\hline Meropenem & $\mathrm{R}$ \\
Nitrofurantoin & $\mathrm{S}$ \\
\hline Tigecycline & $\mathrm{S}$ \\
Doxycycline & $\mathrm{S}$ \\
\hline Tetracycline & $\mathrm{S}$ \\
Ofloxacin & $\mathrm{S}$ \\
\hline Rifampin & $\mathrm{S}$ \\
Piperacillin & $\mathrm{S}$ \\
\hline
\end{tabular}

This case is also interesting because $P$. multocida is typically susceptible to penicillin and cephalosporins. ${ }^{7}$ These drugs are indicated in the treatment of the most frequent form of infection due to $P$. multocida: skin infections following bites or scratches. ${ }^{8}$ Rare penicillin-resistant $P$. multocida strains in human infections have been described and, in these cases, second- and third-generation cephalosporins are recommended for treatment. ${ }^{9,10}$ However, despite resistance to cephalosporins has only been reported in oral first generation antimicrobials belonging to this class, in our case these drugs were ineffective too, thus it was necessary to choose a different therapeutic regimen based on the micro-organism reported susceptibilities to rifampin, TPM/SMX and fluoroquinolones. ${ }^{11}$
2. Donnio PY, Lerestif-Gautier AL, Avril JL. Characterization of Pasteurella spp. strains isolated from human infections. J Comp Pathol 2004;130:137-42.

3. Pond ED, El-Bailey S, Webster D. An unusual case of meningitis. Can J Infect Dis Med Microbiol 2015;26:e62-4.

4. Ebright J, Frey AB, Fairfax MR. Pasteurella multocida infections and bacteremia. Infect Dis Clin Pract 2009;17:102-4.

5. Grillon A, Guillard T, Brasme L, et al. Lethal meningitis due to Pasteurella multocida. Med Mal Infect 2012;42: 374-5.

6. Wade T, Booy R, Teare EL, Kroll S. Pasteurella multocida meningitis in infancy - (a lick may be as bad as a bite). Eur J Pediatr 1999;158:875-8.

7. Noel GJ, Teele DW. In vitro activities of selected new and long-acting cephalosporins against Pasteurella multocida. Antimicrob Agents Chemother 1986;29:344-5.

8. Stevens DL, Bisno AL, Chambers HF, et al. Practice guidelines for the diagnosis and management of skin and soft tissue infections: 2014 update by the infectious diseases society of America. Clin Infect Dis 2014;59:147-59.

9. Giordano A, Dincman T, Clyburn BE, et al. Clinical features and outcomes of Pasteurella multocida infection. Medicine 2015;94:e1285.

10. Naas T, Benaoudia F, Lebrun L, Nordmann P. Molecular identification of TEM-1 beta-lactamase in a Pasteurella multocida isolate of human origin. Eur J Clin Microbiol Infect Dis 2001;20:210-3.

11. Goldstein EJC, Citron DM, Richwald GA. Lack of in vitro efficacy of oral forms of certain cephalosporins, eryth- 